E.L.U.A. 2, 1984, págs. $121-135$

\title{
LOS PREFIJOS VERBALES EN HÚNGARO. A PROPÓSITO DE ALGUNOS ESTUDIOS RECIENTES
}

\author{
NICOLA RAINO \\ (Universidad de Bolonia)
}

0.1. Los estudios de lingüistica general y de lingüística teórica no tienen una larga tradición en Hungría. Como subraya Kiefer Ferenc (1982) en su introducción al volumen Hungarian Linguistics, «until the late fifties Hungarian linguistic life could go on according to tradition" (p. 4). Y esta tradición ha tenido un peso notable, porque, si por un lado ha producido un conjunto enorme de trabajos de gran interés (diccionarios, registros de documentos históricos, etc.), por otro siempre ha imprimido a estos trabajos unos rasgos fuertemente nacionalistas, aislándose del desarrollo de la teoría lingüistica del resto de Europa y conservando una concepción de la lingüística entendida casi exclusivamente como parte de la historia de la cultura y orientada principalmente hacia el análisis de la lengua literaria ' $y$ de los dialectos: pero también en este caso, «it was not the descriptive aspect per se that was important since dialectology, too, served historical purposes», p. 2).

Es a partir del fin de la década de los cincuenta cuando los estudios de carácter descriptivo comienzan a avanzar, y en los años sesenta se amplia el interés también por los análisis de problemas de filosofía del lenguaje, tipologia lingüística y psicolingüística.

' Hay, naturalmente, excepciones. Kiefer recuerda, entre otros, a Gyula Lazicius y a Zoltán Gombocz, pero subraya: «unfortunately, their influence on Hungarian linguistics remained rather sporadic” (p. 3). 
La lingüística húngara ha mantenido desde entonces los contactos con la lingüistica general europeo-norteamericana, hasta los más recientes desarrollos del modelo generativo-transformacional, conservando en todo caso sus propias características, como, por ejemplo, la fuerte atención prestada a los productos más originales de la escuela soviética (sobre todo de tendencia matemática y psicolingüística) y un interés siempre vivo ("tradicional») por la lingüística histórica y por el análisis dialectológico y de área.

0.2. En las páginas que siguen intentaremos dar cuenta de algunos estudios recientes de lingüistas húngaros, enfrentados al análisis de problemas sintácticos, que no sólo proporcionan instrumentos de investigación útiles para la interpretación de la lengua húngara, sino que también se insertan en la discusión general de la estructura de la oración.

En la primera parte se destacarán algunas caracteristicas generales de la estructura oracional del húngaro, y en la segunda se tomará en consideración un problema particular de sintaxis, el movimiento de los llamados "prefijos verbales» en la oración, que recibe una explicación interesante a la luz de nuevas hipótesis teóricas.

1. Tradicionalmente la oración húngara ha sido considerada por los estudiosos como una oración con orden de palabras «libre». Si se abre un conocido manual (Fábián, 1970), se lee a este respecto: “La collocazione delle parole nella frase non è legata a regole severe, come per es. nel tedesco» (p. 15).

Ahora bien, como ha precisado Kiss K. (1981), el orden de palabras en húngaro es considerado «libre» en el sentido de que las palabras de una oración pueden aparecer en un número notable de combinaciones, pero sin embargo también es verdad que no todas estas combinaciones son sinónimas, y además existen algunas que no son posibles ${ }^{2}$. En efecto, el húngaro presenta un orden de palabras libre sólo en lo que respecta al orden de superficie de sujeto, verbo, objeto y de los otros elementos de la estructura de casos ${ }^{3}$. Pero las diversas combinaciones

2 Por ejemplo, el orden «Verbo - Sujeto negado - Objeto».

3 El húngaro sería una lengua no configuracional (véase Chomsky, 1981), en la que el orden de los argumentos es libre y en la que las funciones gramaticales son determinadas no por la configuración de las categorías sintácticas, sino por las terminaciones morfológicas del caso. 
posibles de estos elementos llevan a interpretaciones semánticas diferentes y a modelos de entonación diversos; en otras palabras, las transformaciones del orden de palabras no conservan el significado.

Desde un punto de vista semántico, estas dos oraciones no son sinónimas:
(1) Láttam Jánost
(2) Jánost láttam
(está en cursiva la palabra que lleva el acento principal)

La primera se puede traducir por «he visto a János», y la segunda como "es János a quien he visto».

Estas consideraciones sugieren la idea de que en la oración húngara funciona un orden subyacente, no ligado al sistema de casos. Según Kiss K. (1981), la oración tendria dos posiciones fundamentales a la izquierda del verbo: tópico y foco, y sería posible explicar los cambios del orden de palabras sobre la base de estas dos posiciones.

Precisemos brevemente que por tópico (T) se entiende aqui la posición del elemento inicial de la oración, que es portador de una información conocida, bien por el hablante, bien por el oyente, y que puede ser ocupada por un número arbitrario de complementos verbales, que no llevan el acento de oración y tienen un orden libre (obviamente, siempre en el interior de la misma posición T).

Por foco $(F)$ se entiende, en cambio, aquella posición en la oración entre $T$ y el verbo que es portadora de una información nueva, y puede ser ocupada por un solo complemento verbal, que lleva el acento de oración ${ }^{4}$.

Véase, por ejemplo, una oración muy elemental del húngaro, en la que es evidente todo lo antedicho:
$\begin{array}{ccc}\text { (3) János } & F & V \\ \text { MARIT } & \text { szereti }\end{array}$
János, es Mari + Acusativo a quien él arna
(4) Marit JÁNOS szereti
Mari + Acusativo, es János quien la ama

\footnotetext{
Julia Horvath observa a propósito del acento de oración: “Studying the 'phonological mode' of focusing in English would presumably not be of special interest since in English, apparently, there are no constraints whatever on its occurrence. That is, a grammatical English sentence usually cannot be made ungrammatical by putting emphasis on one of its constituents... In contrast to English, Hungarian manifests a rather interesting system of restrictions on what constituents can be focused where in terms of emphasis" (Horvath, 1976: 177).
} 
La posición $F$, como se ha dicho, lleva siempre el acento de oración cuando está ocupada por el elemento focalizado ${ }^{5}$. En cambio, en caso de que esté vacía, el acento cae sobre el verbo:

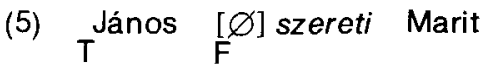

(János ama a Mari + Acusativo)

En la posición posverbal los complementos del verbo no tienen un papel comunicativo relevante: su orden después del verbo (como incluso, se ha dicho, dentro de T) no influye en el significado.

El conjunto de reglas que genera la oración húngara sería, por tanto:

(6) a. $S^{\prime \prime} \rightarrow X^{n *} S^{\prime}$

b. $S^{\prime} \rightarrow X^{n} \quad S^{\circ}$

c. $S^{\circ} \rightarrow V^{n *}$

( $n$ * indica un número arbitrario de categorias máximas).

Según esta regla, $T$ se refiere al conjunto de categorías dominadas inmediatamente por $S^{\prime \prime}$, mientras que $F$ se refiere a la categoria dominada inmediatamente por $S^{\prime}$.

Hay fundadas razones para interpretar $\mathrm{T}$ y $\mathrm{F}$ como posiciones sintácticas distintas una de otra y distintas de las posiciones de los argumentos en S. Ante todo, como se ha dicho, las modificaciones del orden de palabras en $T$ ( $y$ en las posiciones posverbales) no modifican el significado. En segundo lugar, los complementos verbales son sometidos a diversos tipos de restricciones para ocupar $T$ o F; mientras los pronombres interrogativos, por ejemplo, pueden aparecer sólo en F, los pronombres relativos aparecen solamente en la cabeza de T. Además

$s$ En el caso de que la información nueva de la oración sea llevada por un conjunto de complementos verbales, desde el momento en que un solo elemento puede ocupar $F$, solamente uno de los complementos será desplazado a $F$, mientras que los otros permanecerán en la posición posverbal; ejemplo:

- Mit csinálsz?

(¿Qué haces-tú?)

- $_{F}$ [AZ ÚJSAGBAN] olvasok egy cikket

(el periodico-en yo leo un artículo) 
de esto, la extraposición de $\mathrm{S}$ desde la posición $\mathrm{F}$ es obligatoria, mientras es sólo opcional desde T a S. Es posible la elevación de F a F, y de $T$ a $T$, pero no de $S$ a $S^{6}$.

Según el modelo de generación de la oración húngara que hemos propuesto, los nódulos correspondientes a las dos posiciones $\mathrm{T}$ y $\mathrm{F}$ están ocupados por constituyentes posverbales por medio de dos transformaciones de sustitución opcional: topicalización y focalización (que tienen la forma de transformaciones centrales). La transformación de focalización mueve en el nódulo vacio dominado por $\mathrm{S}^{\prime}$ una categoría máxima que sigue al verbo.

En húngaro, además de los complementos modificados por un operador focalizante (es decir, un operador negativo, exclamativo, interrogativo, etc.) hay una segunda clase de complementos que tiene un comportamiento marcado en la focalización: el complemento reducido del verbo. Este último es focalizado en condiciones específicas. Los complementos reducidos del húngaro son el "prefijo verbal" ${ }^{7}$ y los complementos nominales del verbo sin determinante. Ejemplos: «MOZIBA ment János" (al cine ha ido János) “ORVOS lesz János» (médico llega a ser János). Todos estos complementos se caracterizan por su independencia reducida con respecto al verbo, bien sobre el plano semántico (porque forman un elemento único con el verbo, añadiendo solamente algunos rasgos semánticos), bien sobre el plano sintáctico.

En la oración se hallan generalmente próximos al verbo, y con frecuencia antes del verbo, esto es, en posición F. De esta posición pueden ser desplazados sólo por un constituyente enfático o por un constituyente modificado por una focalización (negativa, interrogativa, etc.) ${ }^{8}$. Por ejemplo:

(7) a. A vendégek be viszik a szállodába a bớröndöket Los clientes en - llevan el hotel-en el las maletas (Los clientes llevan al hotel las maletas)

b. Nem a vendégek viszik be a szállodába a böröndöket No los clientes llevan - en el hotel - en el las maletas (No son los clientes quienes llevan al hotel las maletas)

6 A este propósito, véase Kiss K. (1981: 198 ss.).

7 Damos aquí la traducción tradicional del húngaro «igeköt 8 " pero recordamos que Kiss K. ha propuesto para la traducción inglesa la forma "converb", aceptada en Hunyadi (en prensa).

* Así pues, si una oración contiene un complemento reducido, no puede tener $F$ vacio; de hecho, en ausencia de un complemento modificado por un operador-F, el complemento reducido ocupa la posición preverbal $F$. 
La función del prefijo verbal es una característica específica de la lengua húngara, no siendo compartida esta función por todas las lenguas del grupo finougrio ${ }^{9}$. Este elemento se ha desarrollado en tiempos muy antiguos en la historia del húngaro. Según los datos registrados por Soltész K. J. (1959), los seis antiguos prefijos verbales húngaros ("meg», "el», "ki», "be», "fel», "le») son resultado de la evolución de antiguos adverbios que expresaban las principales direcciones de movimiento.

También las lenguas eslavas, como es sabido, tienen un sistema de prefijos verbales que sirven para distinguir parejas de verbos según el aspecto del verbo. De un modo sólo en parte semejante se puede decir que una de las funciones de los prefijos verbales húngaros es la de señalar la diferencia de aspecto ${ }^{10}$. En particular, la función "perfectivizante» es la única función específica del prefijo «meg». Los otros prefijos, como «fel» (arriba), «le» (abajo), «el» (lejos), etc., sirven también para indicar la dirección del movimiento. Sin embargo, además de éstas, los prefijos húngaros también tienen otras funciones; sirven, entre otras cosas, para operar especificaciones léxicas: “a ruhat ki-mosni» (lavar los vestidos) / «a padlot fel-mosni» (lavar el pavimento), y para modificar el significado léxico: «írni» (escribir) / «alá-írni» (firmar).

El prefijo verbal puede ser un síntoma interesante de la evolución tipológica del húngaro. Hoy, para el hablante común, el prefijo verbal es sentido en estrecha relación con el verbo, como un lexema único, y esta opinión es confirmada por muchos estudiosos (por ejemplo, en Dezsö, 1980, oraciones S-pref- $V$ son interpretadas como oraciones SV). En efecto, el prefijo ha llegado a ser, de complemento independiente del verbo, un morfema ligado. Este proceso, que ha tenido inicio probablemente en la primera fase del húngaro antiguo (véase Bárczi, Benkớ, Berrár, 1967) y después ha seguido adelante sobre todo en el plano semántico y fonético. Es posible, por tanto, suponer que la tendencia a interpretar oraciones del tipo S-pref- $V$ como SV sea un síntoma de la tendencia de la lengua a aproximarse al tipo SVO. 184).

Para el uso de prefijos en textos fínicos antiguos, véase A. Sauvageot (1973: $182-$

io "The verbal particle preceding the verb marks the perfective aspect, more precisely a less developed subtype of it: perfective aspectuality, that differs from the perfective aspect of Russian because Hungarian uses the verbal particle to express perfectivization, but has no morphological means like the Russian suffixes to mark imperfectivization " (Dezs 8,1980 : 273). 
El prefijo verbal es, entre los complementos reducidos, el que tiene menor independencia absolutamente: no sólo en el plano sintáctico, sino también en el semántico, puesto que añade solamente al verbo los rasgos de completez y direccióri («olvasni», leer / "el olvasni», terminar de leer), y en el fonético, porque en posición _ $V$ es pronunciado como una única palabra con el verbo.

Su diferencia respecto a los otros complementos verbales es puesta de relieve por el hecho de que, en los casos no marcados, en la posición $F$ no se interpreta como foco (es decir, como el elemento más enfático de la oración) a menos que se le asigne un acento contrastivo. Ejemplo:

(8) János $F^{[L E] ~ m e n t ~ a ~ l e ́ p c s o ́ n, ~ n e m ~ p e d i g ~} F_{F}[F E L] m e n t$

János abajo-fue las escaleras, no arriba-fue (János bajó las escaleras, no subió)

La oración con un prefijo en posición $F(9 b)$ difiere de la que tiene $F$ vacío, sin prefijo, sólo desde el punto de vista de la perfectividad: el prefijo en posición $F$ en (9b) hace perfectivo el verbo:

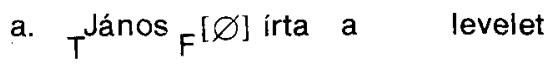
János escribió la carta
(János estaba escribiendo la carta)
b. $T^{\text {János }} F^{[M E G] \text { írta a levelet }}$ János arriba escribió la carta (Juan escribió/ha escrito la carta)

En cambio, cuando el prefijo se encuentra en $S$ no tiene función aspectual; tiene función aspectual $F$ vacio, con el prefijo a la derecha del verbo:

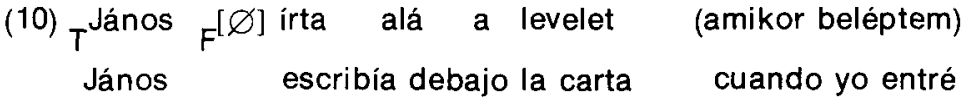 (János estaba firmando la carta cuando yo entré)

que en este caso expresa el aspecto continuo del verbo, mientras que en (11) expresa tiempo indefinido: 


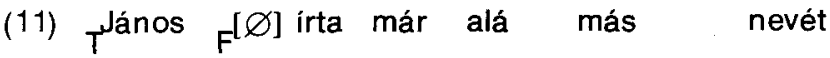 János escribió ya debajo de otro el nombre (János ha firmado ya con el nombre de otro)

Aun siendo, como se ha dicho, un complemento reducido, que constituye una unidad semántica y fonética con el verbo, el prefijo verbal es afectado por las reglas sintácticas de la lengua del mismo modo que un complemento verbal independiente. Veamos, pues, las principales posiciones del prefijo verbal en el interior de la oración:

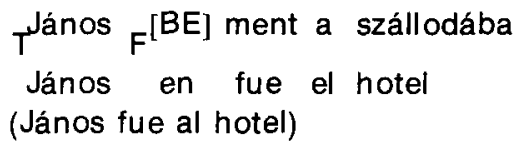

En este caso, que es el menos marcado, el prefijo ha sido desplazado a $F$, en donde se comporta como operador de perfectividad, y su posición es la de cualquier complemento verbal independiente focalizado. Por ello puede, normalmente, ser precedido por la partícula negativa:

$T^{\text {János nem }} F_{F}[B E]$ ment a szállodába, hanem $F_{F}[B E]$ rohant János no en fue el hotel pero en se precipitó (János no fue al hotel, sino se precipitó a él)

o bien es seguido por ella:

(14)

$T^{\text {János }} F^{[B E]}$ nem ment a szállodába

János en no fue el hotel

(János no fue al hotel de ningún modo)

También en las oraciones imperativas el prefijo se encuentra en posición F:

$$
\begin{aligned}
& T^{\text {János }} \quad F^{[B E] \text { menjen a szállodába }} \\
& \text { János en ir+imperativo el hotel } \\
& \text { (János debe ir al hotel en cualquier modo) }
\end{aligned}
$$


En los ejemplos siguientes el prefijo permanece en la posición generada por la base, es decir, a la derecha del verbo. Permanece en $S$ porque el tiempo indefinido del verbo es expresado con $F$ vacio:
(16) János $_{F^{[\varnothing]} \text { ment már be a szállodába }}^{[\varnothing]}$
János fue ya en el hotel
(János ha ido ya al hotel)

o bien porque la posición $F$ está ocupada por otro complemento focalizado:

(17) $T^{[\varnothing]} F^{[J A ́ N O S] ~ m e n t ~ b e ~ a ~ s z a ́ l l o d a ́ b a ~}$ János fue en el hotel

(Fue János quien fue al hotel)

Incluso en el caso de oraciones negativas o imperativas el prefijo puede ir detrás del verbo, en posición no marcada:

(18)

$$
\begin{aligned}
& T^{\text {János }} F^{[\varnothing]} \text { nem ment be a szállodába } \\
& \text { János no fue en el hotel } \\
& \text { (János no fue al hotel) }
\end{aligned}
$$

$$
\begin{aligned}
& \text { (19) } \underset{T}{\text { János }} \mathrm{F}^{[\varnothing] \text { menjen }} \text { be a szállodába } \\
& \text { János ir+imperativo en el hotel } \\
& \text { (János debería ir al hotel) }
\end{aligned}
$$

(como se ha visto en 14 y 15 , es posible el orden con el prefijo a la izquierda del verbo, pero está marcado al tener una fuerte carga de énfasis).

El prefijo también puede ser topicalizado:

(20) $T^{\mathrm{Be}} \mathrm{F}^{[\mathrm{JAN} O S] \text { ment a szállodába }}$

en János fue el hotel

(En cuanto a ir alli, fue János quien fue al hotel)

o desplazado por la elevación: 
(21) János $F^{[B E]}$ akar menni a szállodába

János en quiere ir el hotel

(János quiere ir al hotel)

(en este caso hay elevación de una oración de infinitivo en $F$ ).

Según el modelo elaborado por Kiss (1981), la estructura siguiente:

(22)

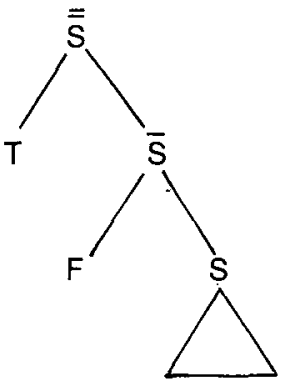

representa la estructura sintáctica de los tipos de oración en húngaro. Sin embargo, hay un tipo de oración que no parece pueda ser generada con este esquema, según sostiene Kiss (en prensa): aquel en el cual se encuentran unas expresiones cuantificadas a ta izquierda del verbo. La oración de (23) no puede ser generada por las reglas sintácticas de base del húngaro:

$$
\begin{aligned}
& \mathrm{T}^{\text {[János] mindig }} \mathrm{F}^{[\mathrm{BE}] \text { megy a szállodába }} \\
& \text { János siempre en va el hotel } \\
& \text { (János siempre va al hotel) }
\end{aligned}
$$

La expresión cuantificada en sentido universal positivo mindig no puede ser colocada en $F$, donde debe estar el prefijo, ni en $T$, primero porque lleva el énfasis, y después porque, si fuese parte de $T$, podría ser invertida con el otro elemento de $T$, lo que no es posible:

$$
\begin{gathered}
T^{*}[\text { Minding János }] \\
F^{[B E]} \text { megy a szállodába } \\
\text { Siempre János en va el hotel }
\end{gathered}
$$

Según Kiss (en prensa), es necesario suponer una tercera posición 
$Q$ (=cuantificador) a la izquierda del verbo, entre $T$ y $F$, que contenga las expresiones cuantificadas, una o más:
(25) $T^{\text {János }} \mathrm{Q}^{\text {[mindig mindent] }} \mathrm{F}$ EL olvas
János siempre todo PREFIJO lee
(János siempre lee todo)

Las expresiones cuantificadas en sentido universal (positivas o negativas) pueden aparecer sólo en $\mathrm{Q}$, en posición preverbal (si, por el contrario, se encuentran a la derecha del verbo, su comportamiento es el de cualquier otro complemento):
(26) $T^{\text {János }} Q^{\text {[semmit] }} F$ EL nem olvasott
János nada PREFIJO no leer (pasado)
(János no ha leído nada)

Las expresiones con cuantificación positiva (pero no universal) pueden aparecer, bien en $F$, bien en $Q$ :

\begin{tabular}{|c|c|c|c|}
\hline $\begin{array}{l}\text { (27) János } \\
\text { János } \\
\text { (János }\end{array}$ & $\begin{array}{c}Q^{[\text {sok könyvet] }} \\
\text { muchos libros } \\
\text { ha leido muchos libro }\end{array}$ & $\begin{array}{l}\text { FL }^{\text {EL }} \\
\text { PREFIJO } \\
\text { S) }\end{array}$ & $\begin{array}{l}\text { olvasott } \\
\text { leer (pasado) }\end{array}$ \\
\hline $\begin{array}{l}\text { 8) János } \\
\text { János } \\
\text { (János }\end{array}$ & $\begin{array}{l}\mathrm{F}^{\text {[SOK KONYVET] }} \\
\text { muchos libros } \\
\text { ha leído muchos libro }\end{array}$ & $\begin{array}{l}\text { olvasott } \\
\text { leer } \\
\text { s) }\end{array}$ & $\begin{array}{l}\text { el } \\
\text { PREFIJO }\end{array}$ \\
\hline
\end{tabular}

Mientras las expresiones con cuantificación universal no pueden aparecer nunca en $\mathrm{F}$, aquéllas con cuantificación sólo positiva pueden ocupar $\mathrm{F}$ tanto más fácilmente cuanto más alejadas estén de la universalidad o de la generalidad.

Se da el caso de expresiones con alto grado de generalidad en posición $\mathrm{F}$, pero solamente en situaciones que expresan un contraste explícito:
$T^{\text {János }} F^{\text {[GYAKRAN] ijed }}$
meg, Péter ${ }_{F}$ [RITKAN]
János a menudo se asusta arriba, Péter raramente (János a menudo se asusta, Péter raramente) 
Uno de los problemas más intrincados, por el que se han interesado ampliamente los investigadores, es precisamente el de explicar el comportamiento diverso del prefijo verbal en presencia de expresiones que contienen una cuantificación o valoración positiva (expresiones tradicionalmente llamadas inclusivas- "összefogladó") y en presencia de expresiones con cuantificación o valoración negativa (exclusivas- «kirekesztó"). Por ejemplo:

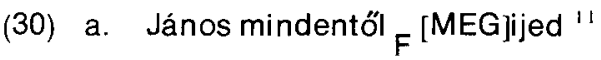

János todo-de arriba se asusta

(János se asusta de todo)

b. János SEMMITÓL $F[\varnothing]$ nem ijed meg

János nada-de no se asusta arriba

(János no se asusta de nada)

(31) a. János mindig $F$ MEG ijed

János siempre arriba se asusta

(János siempre se asusta)

b. János SOHA $F[\varnothing]$ nem ijed meg

János nunca no se asusta arriba

(János nunca se asusta)

(32) a. János gyakran $F^{[M E G]}$ ijed

János a menudo arriba se asusta

(János a menudo se asusta)

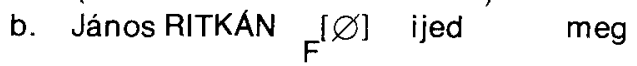

János raramente se asusta arriba

(János raramente se asusta)

"Es posible probar que tampoco las expresiones con cuantificador negativo aparecen en $F$, que permanece vacio: de hecho, en este caso es posible insertar un complemento verbal entre aquellas expresiones y el verbo, esto es, en la posición de $F$ vacio; ejemplo:

- János semmitol ${ }_{F}[\varnothing]$ nem ijed meg

János nada-de no se asusta arriba

- János semmitol $[$ [MEG] nem ijed

János nada-de arriba no se asusta 
Lo que hasta ahora se había observado es que el prefijo debe hallarse a la izquierda del verbo en presencia de una expresión «inclusiva»; en cambio, a la luz de las observaciones arriba citadas, es claro que es otra la restricción, esto es, que la proposición inclusiva no debe preceder inmediatamente al verbo, debiendo ocupar.la posición Q. De hecho, antes del verbo, en $\mathrm{F}$, puede haber cualquier otro complemento del verbo; por tanto, no sólo el prefijo verbal. Ejemplo:

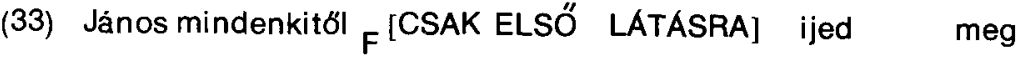 János cada uno-de sólo primero vista-a (János se asusta de cualquiera sólo a primera vista)}

y esto también es válido para oraciones en las que el verbo no tenga de hecho el prefijo verbal:
(34) Jánosnak
mindig $_{F}[$ KÉT TALALÁTA]
van a lottón
János +Dativo siempre dos números suyos son la lotería-en (János siempre tiene dos números en la lotería)

De estas consideraciones resulta evidente que no es el prefijo verbal lo que se comporta de modo irregular en presencia de una expresión cuantificada positiva, sino que el comportamiento particular lo presenta la expresión cuantificada, haya o no un prefijo verbal.

Las observaciones hechas hasta aquí tienen como único objeto introducir algunos problemas, presentando algunas posibles direcciones de investigación. Ciertamente, el tema tratado es uno de los más interesantes que se propone la lingüistica húngara actual, y ya están abiertos otros sectores de investigación. Uno de muy alto interés, entre otros, es el relativo a las implicaciones semánticas de ciertas funciones de los prefijos verbales en la oración, como la de expresar las condiciones de determinación de algunos elementos (sobre todo sujeto y objeto), tema recientemente afrontado por Vörös J. $(1973,1980)$.

Otro problema, muy complejo pero de gran interés, que aquí ha sido solamente apuntado, es el de la relación prefijos verbales - aspecto verbal replanteado en un original análisis por Kiefer Ferenc en su ensayo "The aspectual system of Hungarian» (Kiefer, 1982). Este trabajo, que coloca sobre nuestras bases el análisis del aspecto verbal húngaro, desarrolla un análisis puntual del comportamiento del prefijo "meg" (cuyo único significado es: «it makes a situation perfective», p. 322) para intentar responder a la pregunta "Are prefixes predictable?". Las 
respuestas de Kiefer son altamente convincentes y permiten estimular aún más la investigación sobre este tema.

(Traducido del italiano por Tomás Albaladejo)

\section{Referencias bibliográficas}

Bárczi, G.; Benkớ, L.; Berrár, J. (1967), A magyar nyelv története, Budapest, Tankönyvkiadó.

Chomsky, N. (1981), Lectures on Government and Binding, Dordrecht, Foris.

Dezsó, L. (1980), «Word Order, Theme and Rheme in Hungarian and the Problems of Word-Order Acquisition», en: Dezs 6 , L.; Nemser, W. (eds.) (1980), pp. 245-298.

- Nemser, W. (eds.) (1980), Studies in English and Hungarian Contrastive Linguistics, Budapest, Akadémiai Kiadó.

Fábián, P. (1970), Manuale della lingua ungherese, Budapest, Tankönyvkiadó.

Horvath, J. (1976), «Focus in Hungarian and the $\bar{X}$ Notation», en: Linguistic Analysis, 2, 2, pp. 175-197.

- (1980), "Core Grammar and a Stylistic Rule in Hungarian Syntax», en: NELS $\bar{X}, 9$, pp. 237-255.

Hunyadi, L. (en prensa), "The Syntax and Semantics of Topic and Focus: Another Approach», en: Acta Linguistica Academiae Scientiarum Hungaricae.

Kiefer, F. (1967), On Emphasis and Word-Order in Hungarian, Bloomington, Indiana University Press.

- (1982), "The Aspectual System of Hungarian», en: Kiefer, F. (ed.), Hungarian Linguistics, Amsterdam, J. Benjamins, 1982, pp. 293329.

Kiss, K. (1981), "Structural Relations in Hungarian, a 'Free' Word Order Language», en: Linguistic Inquiry, 12, pp. 185-213.

- (en prensa), "The Problem of the Verbal Prefix. Quantifier Preposing".

Perrot, J. (1966), «Adalékok a 'meg' igeköt $\delta$ funkciójának vizsgálatához a mai magyar nyelvben", en: Nyelvtud. Ert., 52, sz.

Sauvageot, A. (1973), L'élaboration de la langue finnoise, París, Klincksieck.

Soltész, K. (1959), Az ôsi magyar igekötơk, Budapest, Akadémiai Kiadó. 
Szabolcsi, A. (1981), "The Semantic of Topic-Comment Articulation", en: Groenendijk; Janssen; Stokhof (eds.), MC TRACT 136. Formal Methods in the Study of Language.

Vörös, J. (1973), «Az igekötő helyének mondatjelentés-megkülönböstetớ szerepe», en: Magyar Nyelvớr, 4, pp. 416-422.

- (1980), "Syntactic Structures of Hungarian Verbal Particles and their English Equivalents", en: Dezs6", L.; Nemser, W. (eds.) (1980), pp. 299-317. 